\title{
Highly Stable Multi-Anchored Magnetic Nanoparticles for Optical Imaging within Biofilms
}

\author{
R. C. Stone, ${ }^{a}$ B. D. Fellows, ${ }^{a}$ B. Qi, ${ }^{a}$ D. Trebatowski, ${ }^{b}$ B. Jenkins, ${ }^{c}$ Y. Raval, ${ }^{c}$ T.R. Tzeng, ${ }^{c}$ T. F. Bruce, ${ }^{c, e}$ T. \\ Mcnealy, ${ }^{c, d}$ M.J. Austin, ${ }^{\mathrm{f}}$ T. C. Monson, ${ }^{\mathrm{f}}$ D. L. Huber, ${ }^{\mathrm{f}}$ and O. T. Mefford. ${ }^{a, d}$
}

KEYWORDS magnetic nanoparticles, heterobifunctional,multi-anchored, polyethylene oxide, click chemistry, biofilms, imaging, nitroDOPA, fluorescence.

\begin{abstract}
Magnetic nanoparticles are the next tool in medical diagnoses and treatment in many different biomedical applications, including magnetic hyperthermia as alternative treatment for cancer and bacterial infections, as well as the disruption of biofilms. The colloidal stability of the magnetic nanoparticles in a biological environment is crucial for efficient delivery. A surface that can be easily modifiable can also improve the delivery and imaging properties of the magnetic nanoparticle by adding targeting and imaging moieties, providing a platform for additional modification. The strategy presented in this work includes multiple nitroDOPA anchors for robust binding to the surface tied to the same polymer backbone as multiple poly(ethylene oxide) chains for steric stability. This approach provides biocompatibility and enhanced stability in fetal bovine serum (FBS) and phosphate buffer saline (PBS). As a proof of concept, these polymer-particles complexes were then modified with a near infrared dye and utilized in characterizing the integration of magnetic nanoparticles in biofilms. The work presented in this manuscript describes the synthesis and characterization of a nontoxic platform for the labelling of near IR-dyes for bioimaging.
\end{abstract}

\section{Introduction}

Functional magnetic nanoparticles have become an important research focus for use in biomedical applications. These applications include magnetic hyperthermia,[1-4] magnetic resonance imaging,[1, 3, 5, 6][1,3,5,6] cell separation,[5-7]and remediation of biofilms.[8] The small size and manipulation of magnetic properties of these materials also have potential for non-invasive diagnostics and treatments to improve quality of life for patients.

There are a variety of ways to create functional magnetic nanoparticles, but the fundamental designs of these materials stay the same. Generally, in each individual nanoparticle, the core material is magnetic and is coated with a hydrophilic material that is either physically or chemically adsorbed on to the surface. The core can be made up of an assortment of magnetic materials, including iron platinum, cobalt oxide, and iron oxides.[7] Because these materials are generally synthesized using thermal decomposition methods, they have hydrophobic polymer ligands that are only dispersible in organic solvents. They must be modified with hydrophilic materials for biomedical applications. There are a variety of hydrophilic surface designs that have been investigated to create hydrophilic magnetic nanoparticles, including dextran, pluronics, and polyethylene oxide (PEO).[7, 9]

Magnetic nanoparticles for biomedical applications, like any pharmaceutical delivery vehicle, have power in numbers. The ultimate goal is to have a high accumulation in the affected area without using high dosages that may result in side effect for the patient. This can be accomplished in magnetic nanoparticle composites by designing polymer ligands that increases the bioavailability of the particles in the circulatory system,[7, 9] as well as taking advantage of the particles' surface to target certain cells by exploiting over expressed receptors.[7, 10]

Designing polymer ligands for biological targeting and imaging applications can be exhaustive and the reactions can be inefficient. Functionalization of the nanoparticles generally requires harsh solvents that must be completely removed before any biological interaction.[11] Click chemistry provides a biologically friendly conjugation method to create strong and chemically stable linkages. Originally developed for facile protein modification, this method was later used to efficiently modify polymers.[12]The most common of the click reactions is the Huisgen 1,3-dipolar cycloaddition between an alkyne and an azide.[13]

We have previously reported synthesis of monoanchor systems consisting of a catechol binding group on one end and clickable alkyne on the other end.[14, 15]. This provides a versatile platform for additional modification, as we and others have demonstrated having multiple anchors (i.e., f>3) prevents salts and 
proteins from disrupting the steric stability of these systems. To provide this 'multidentate' binding to the surface, we adapt chemistry for the modification of polyacrylic acid (PAA) with catechol amines and PEO, which has been successfully demonstrated by Wang and Na.[10, 16] Here, we report the synthesis and characterization of a magnetic nanoparticle composite comprised of an iron oxide core and a functional PEO complex with enhanced colloidal stability in biological environments, due to multi-anchoring of catechol groups to the surface. This polymer-particle complex can be tailored for a variety of biomedical applications using click chemistry. To illustrate the enhanced stability of the aforementioned particle, it was exposed to a variety of simulated biological environments at body temperature and compared to a mono-anchored particle of similar size and composition.

To demonstrate the versatility of our platform, we utilized dye labeled versions of these materials for observing how polymer-coated magnetic nanoparticles interact with Legionella biofilms. We have recently reported changes in the morphology and decrease of biomass in Legionella pneumophila biofilms after exposure to low concentrations of magnetite nanoparticles $\left(\mathrm{Fe}_{3} \mathrm{O}_{4} \mathrm{NPs}\right)$,[8] and sought an effective method for the visualization of the fate of these materials. Previous efforts for the visualization of iron oxide particles in cellular environments have included staining with Prussian blue,[17] PET labels,[18] and IR dyes,[19] MRI, and others. Despite these advances, visualization of the particles within biological samples remains a challenge due to the highly absorptive nature of iron oxide materials. Several different strategies have been proposed throughout the years.[20] Here, in we use a polymer brush linker to extend the dye away from the iron oxide particle. Demonstrating the effectiveness of this technique, the multi-anchored particles were modified with Cy5.5 and imaged in biofilms to observe the fate of these nanoparticles. This dye was selected as excitation and emission are in the near infrared (NIR) region, from 650 to $900 \mathrm{~nm}$. In this region, the absorbance and autofluorescence of biological media is low providing better energy transfer through the tissue.[20] In this work, we show a magnetic nanoparticle system that has enhanced biological stability and has been modified with a NIR dye, which can be used to observe the nanoparticle fate within a biofilm using NIR imaging.

\section{Experimental Section}

\section{Materials and Characterization}

Tetrahydrofuran (THF; B.D.H. ACS grade) was purified by reflux over sodium metal (Aldrich Chemistry; sodium lump in kerosene 99\%) and benzophenone. Bromophenol blue (Sigma Aldrich), hydrochloric acid (HCl; J.T. Baker, 1N volumetric solution), ethylene oxide (EO; Aldrich Chemistry), dichloromethane (DCM; EMD Millipore Chemicals, HPLC grade), chloroform (B.D.H.), diethyl ether (DEE; Macron Chemicals), triethyl amine (TEA; Alfa Aesar 99\%), 4-(dimethylamino) pyridine (DMAP; Fluka Analytical), succinic anhydride (SA; Alfa Aesar 99\%), N,N'-dicyclohexyl carbodiimide (DCC; Thermo Scientific), N-hydroxysuccinimide (NHS; Acros Organics 98+\%), Cy5.5 azide (Lumiprobe 95\%), poly(acryclic acid) 1800MW (PAA; Aldrich), copper (II) sulfate (Sigma Aldrich), (+)sodium L-ascorbate (Sigma Aldrich), chloroform-D $\left(\mathrm{CDCl}_{3} ; \mathrm{D}, 99.8 \%\right.$, Cambridge Isotope Laboratories Inc.), potassium bis(trimethylsilyl)amide solution (1M THF, Aldrich), propargyl bromide solution (80\% in toluene; Fluka), sodium hydride (NaH; Aldrich), dimethylformamide (DMF; 99.8\%, extra dry over molecular sieves; Sigma Aldrich), methanol (absolute ACS reagent grade; Ricca Chemical Company), and Spectrum Spectra/Por ${ }^{\circledR}$ molecular porous membrane tubing, MWCO of 1,000 and 12-14,000 were all used as received. Materials used for cell viability studies were as follows: 96 well plates were purchased from Corning, L-929 cells $\left(\right.$ ATCC $^{\circledR}$ CCL-1 ${ }^{\mathrm{TM}}$ ) and Eagle's Minimum Essential Medium (EMEM) were purchased from American Type Culture Collection (ATCC), phosphate buffer solution (PBS) from Sigma Aldrich was autoclaved prior to usage, CellTiter $96^{\circledR} \mathrm{AQ}_{\text {ueous }}$ One Solution Cell Proliferation Assay kit (MTS) from Promega was used as received.

All the polymers were characterized using hydrogen nuclear magnetic resonance $\left({ }^{1} \mathrm{HNMR}\right)$ on a Joel ECX-300 using deuterated chloroform as the solvent. The thermal properties of the particles were measured using a Hi-Res 2950 thermogravimetric analyzer (TGA) from TA Instruments, by first driving off any water at $100{ }^{\circ} \mathrm{C}$ for 30 minutes, followed by a ramp to $800{ }^{\circ} \mathrm{C}$ at a rate of $20{ }^{\circ} \mathrm{C} / \mathrm{min}$. The photoluminescence of the modified particles were characterized using a Horiba Jobin-Yvon MicroHR spectrophotometer, excitation at $655 \mathrm{~nm}$ and collected from 665 to $800 \mathrm{~nm}$. Transmission electron microscopes (TEM) micrographs were acquired at an accelerating voltage of 300KV on a Hitachi H-9500 and the mean size was acquired by averaging the diameter of 181 particles using FoveaPro image analysis software. Particle hydrodynamic diameter was measured using dynamic light scattering (DLS) on a 
Malvern Zetasizer Nano ZS (Model:ZEN3600). DC magnetization measurements were completed on a Quantum Design MPMS-7 SQUID magnetometer using samples of $1 \mathrm{wt} \%$ iron or less. Temperature sweeps were per- formed by zero-field cooling the sample and then measuring the magnetic moment as a function of temperature under the influence of a weak magnetic field $(1 \mathrm{mT})$ during warming and subsequent cooling. Precise masses for each sample were determined destructively, by forming the phenanthroline/ $/ \mathrm{Fe}^{+}$complex, and spectrophotometrically quantifying the concentration of a known dilution.

\section{Biological Characterization}

The potential cytotoxicity of the multi-anchored particles was determined by performing MTS assay on L929 cells.[15, 21] Briefly, the cells were grown on $50 \mathrm{~cm}^{2}$ tissue-culture flask (Corning, NY) in the presence of EMEM at $37^{\circ} \mathrm{C}$ in a humidified atmosphere of $5 \% \mathrm{CO}_{2}$ and $95 \%$ air. EMEM was supplemented with $2 \mathrm{mM}$ L-Glutamine, non-essential amino acids, FBS (final concentration - 10\%), $100 \mathrm{UI} / \mathrm{ml}$ penicillin $\mathrm{G}$, and $100 \mu \mathrm{g} / \mathrm{mL}$ streptomycin. $1.5 \times 10^{4}$ cells/well were seeded (in triplicates) in 96-well culture-plates at $37{ }^{\circ} \mathrm{C}$ in a humidified atmosphere of $5 \% \mathrm{CO}_{2}$ and $95 \%$ air. After 24 hours, varying concentration of particles were added to the cells and incubated for further 24 hours. Next day, MTS assay was performed according to manufacturer's protocol and the plate was read at $490 \mathrm{~nm}$ optical density to measure the absorbance of the formazan product using a microplate reader (Thermo Scientific Multiskan ${ }^{\mathrm{TM}}$ FC). Also, wells containing only particles without any cells/ MTS reagent were used as blanks to account for particle interference, if any.

Legionella pneumophila biofilms were established over five days in a six well plate on sterile glass coverslips. Mature biofilms were exposed to $1 \mu \mathrm{g} / \mathrm{L}$ Cy5.5-tagged iron oxide nanoparticles (IONPs). After $48 \mathrm{~h}$, biofilms were washed with 1X PBS then allowed to air dry. Biofilms were fixed in $4 \%$ paraformaldehyde and gently washed with 1X PBS. After fixation, biofilms were stained with $3 \mu \mathrm{M}$ Syto11 nucleic acid stain from Invitrogen. Mowiol mounting media was added to a depression slide and the dried coverslip was carefully placed over this filled depression. The edges of the coverslip were sealed with Twinsil ${ }^{\circledR}$ and allowed to harden for 5-10 minutes. The biofilms were imaged on a Leica SR GSD 3D, Super Resolution Ground State Depletion 3D Microscope (Leica Microsystems, Buffalo Grove, IL) using a 160 X TIRF objective with a cylindrical lens (Leica HCX PL APO 160x/ 1.43 NA, oil immersion).

\section{Synthetic Procedures}

\section{Synthesis of Iron Oxide Nanoparticles.}

The $7.2 \mathrm{~nm}$ magnetic nanoparticles used for both polymer-particle composites where synthesized using thermal decomposition of iron(III) acetylacetonate (2 mmol), 1,2-hexadecanediol (10mmol), olylamine (4 $\mathrm{mmol})$, benzyl ether $(20 \mathrm{ml})$.[5, 6, 16, 22] The reaction was brought to reflux for 30 min under a nitrogen head. The particles were purified by precipitation using ethanol and characterized using TEM and DLS.

\section{Synthesis of NitroDOPA.}

In a $50 \mathrm{ml}$ round bottom flask, equipped with a stir bar, $30 \mathrm{ml}$ of DI water was cooled in a brine salt bath to $0{ }^{\circ} \mathrm{C}$ before charging with sodium nitrite $(1.52 \mathrm{~g}, 17.88 \mathrm{mmol})$ and L-3,4-dihydroxyphenylalanine (LDOPA) (1.97 g, $9.99 \mathrm{mmol})$ and allowed to cooled. While stirring, $0.92 \mathrm{ml}(90.23 \mathrm{mmol})$ of sulfuric acid was added dropwise to form an orange precipitate. Once all of the acid was added, the solution was stirred overnight. The product was collected by vacuum filtration, washed with methanol, and dried in a vacuum oven.

${ }^{1} \mathrm{HNMR}\left(300 \mathrm{MHz}, \mathrm{CDCl}_{3}\right), \delta(\mathrm{ppm}): 6.1(\mathrm{CH}$, ring, nitroDOPA), $6.8(\mathrm{CH}$, ring, nitroDOPA), 1.8 and 2.4 (ring- $\mathrm{CH}_{2}-\mathrm{CH}_{2}-\mathrm{C}$ ).

\section{Synthesis of Protected Amine-PEO-OH.}

The procedure used is based on previous work done by Kim et al.[5,6,21,22] Briefly, ethylene oxide was polymerized using potassium bis(trimethylsilyl)amide as the initiator and terminated by exposure to air. The polymer was purified by multiple precipitations in DEE and collected by filtration. (MW was determined to be 6000 via ${ }^{1} \mathrm{H}$ NMR.)

\section{Synthesis of Protected Amine-PEO-Alkyne.}

Protected Amine-PEO-OH (2.0 g, $0.33 \mathrm{mmol})$ and sodium hydride (32 $\mathrm{mg}, 1.32 \mathrm{mmol})$ was charged to a $100 \mathrm{ml}$ round bottom flask equipped with a stir bar. The flask was the sealed with a septum and purge with 
nitrogen for 30 seconds. THF was added via syringe and the reaction was brought to $0{ }^{\circ} \mathrm{C}$ using an ice bath. Propargyl bromide $(0.15 \mathrm{~g}, 1.32 \mathrm{mmol})$ was slowly added via syringe, over 30 minutes. All the reactants were left to stir for another 30 minutes at $0{ }^{\circ} \mathrm{C}$. The flask was then removed from the ice bath and brought to room temperature to react for 24 hours in the absence of light. The final solution was filtered to remove excess sodium hydride and precipitated with DEE twice to collect polymer.

${ }^{1} \mathrm{HNMR}\left(300 \mathrm{MHz}, \mathrm{CDCl}_{3}\right.$ ), $\delta(\mathrm{ppm}): 3.56\left(\mathrm{~m}, \mathrm{C}_{2}-\mathrm{C}_{2}-\mathrm{O}\right.$ ), 2.43 (s, -O-CH$\left.{ }_{2}-\mathrm{C} \equiv \mathrm{C} \underline{H}\right), 4.19$ (s, $-\mathrm{O}-\mathrm{C}_{2_{2}}{ }^{-}$ $\mathrm{C} \equiv \mathrm{CH}$ ), 2.75 (m, (TMS) $\left.{ }^{2}-\mathrm{N}-\mathrm{CH}_{2}-\right) 1.19$ (m, TMS).

\section{Synthesis of Amine-PEO-Alkyne.}

The deporotection of the amine was accomplished by dissolving the polymer $(1.8 \mathrm{~g}, 0.30 \mathrm{mmol})$ into methanol and then slowly adding 20 drops of $1 \mathrm{M} \mathrm{HCl}$ and stirred overnight under a nitrogen head. The product was precipitated with DEE (2x) and solid was collected. The cleavage of the bis(trimethylsilyl) (BTMS) group was confirmed by HNMR.

${ }^{1} \mathrm{HNMR}$ (300 MHz, CDCl3), $\delta$ (ppm): 3.65 (m, CH2-CH2-O), 2.44 (s, -O-CH2-C $\equiv \mathrm{CH}$ ), 4.19 (s, -O-CH2$\mathrm{C} \equiv \mathrm{CH})$

\section{Synthesis of PAA-PEO-Alkyne.}

In a $100 \mathrm{ml}$ round bottom flask equipped with a stir bar and condenser, Amine-PEO-Alkyne (0.8 g, 0.14 mmol) was dissolved in $40 \mathrm{ml}$ of THF and brought to $0{ }^{\circ} \mathrm{C}$. DCC $(0.03 \mathrm{~g}, 0.14 \mathrm{mmol})$ was then added and stirred for 30mins under constant nitrogen purge. Finally PAA (0.05 g, $0.03 \mathrm{mmol})$ and DMAP (0.003 g, $0.03 \mathrm{mmol}$ ) was added and brought to reflux at $70{ }^{\circ} \mathrm{C}$ for 2 days. After two days, the solution was filtered to remove any undissolved reactants and precipitated with DEE. The polymer was then dissolved in DCM and precipitated with DEE (2X) and the final solid was characterized by HNMR.

${ }^{1} \mathrm{HNMR}$ (300 MHz, CDCl3), $\delta$ (ppm): 3.56 (m, CH2-CH2-O), 2.43 (s, -O-CH2-C $\equiv \mathrm{CH}$ ), 4.20 (s, -O-CH2$\mathrm{C} \equiv \mathrm{CH}),(\mathrm{b}, 1.20-2.25$, PAA).

\section{Synthesis of NitroDOPA-PAA-PEO-Alkyne.}

To a $100 \mathrm{ml}$ flame dried, round bottom flask equipped with a stir bar, PAA-PEO-Alkyne $(0.4 \mathrm{~g}, 0.0015$ mmol) was dissolved in DMF and brought to $0{ }^{\circ} \mathrm{C}$ using an ice bath. EDC (0.002 g, $\left.0.013 \mathrm{mmol}\right)$ and TEA $(2.0 \mu \mathrm{L}, 0.015 \mathrm{mmol})$ were both added while purging with nitrogen and stirred at $0{ }^{\circ} \mathrm{C}$ for 30 mins. The final reactants, nitroDOPA (3.0 mg, $0.012 \mathrm{mmol}$ ) and DMAP $(0.3 \mathrm{mg}, 0.003 \mathrm{mmol})$, were added under a constant nitrogen purge and stirred for another $30 \mathrm{mins}$. Finally, the reaction was removed from the ice bath and brought to $80{ }^{\circ} \mathrm{C}$ for 5 days. The purification was accomplished by bringing the reaction to room temperature and filtering out any solids. The polymer was then precipitated with DEE and the solid was dissolved in chloroform. Vacuum filtration was then used to remove any excess nitroDOPA. The filtrate was collected and polymer was collected by precipitation in DEE (2x). The polymer was then dissolved in DI water and dialyzed using 1000 MWCO dialysis membrane. Product was confirmed by ${ }^{1} \mathrm{HNMR}$ and FTIR.

${ }^{1} \mathrm{HNMR}$ (300 MHz, CDCl 3 ), $\delta$ (ppm): 3.62 (m, $\mathrm{C}_{2}-\mathrm{C}_{2}-\mathrm{O}$ ), 2.43 (s, -O- $\mathrm{CH}_{2}-\mathrm{C} \equiv \mathrm{C} \underline{H}$ ), 4.19 (s, -O-C $\underline{H}_{2}-$ $\mathrm{C} \equiv \mathrm{CH}$ ), (b, 1.20-2.25, PAA). NitroDOPA: 6.12 and 6.70 (s, $\mathrm{CH}$ in ring).

\section{Particle Modification.}

NitroDOPA-PAA-PEO-Alkyne (0.2 g) was dissolved in $10 \mathrm{ml}$ of chloroform. $7.2 \mathrm{~nm}$ particles in hexane (1 $\mathrm{ml}, 2 \mathrm{mg} \mathrm{ml}^{-1}$ ) were slowly added over 30 minutes while using sonication. Once all the particles were added, the solution was stirred overnight. The particles were then precipitated with hexane and dispersed in $5 \mathrm{ml}$ of ethanol to dissolve any of the displaced oleylamine and precipitated with hexane to collect the polymer-modified particles. The particles were then dispersed in DI water and dialyzed for 2 days using 1214,000 MWCO dialysis membrane. The particles where then characterized by DLS and TGA.

\section{Modifying Particles with Dye}

To $10 \mathrm{ml}$ of DI water, $0.5 \mathrm{ml}$ of the polymer modified particles $\left(0.7 \mathrm{mg}\right.$ of modified particles $\left.\mathrm{ml}^{-1}\right)$ was added. Azido Cy5.5 (0.003 mmol) was added to the solution followed by copper (II) sulftate $(0.013 \mathrm{mmol})$ and sodium ascorbate $(0.003 \mathrm{mmol})$. The reaction was then done in the absence of light at $28{ }^{\circ} \mathrm{C}$ for 12 hours. The particles where then subjected to dialysis to remove any excess dye and catalyst.

\section{Results and Discussion}


Iron oxide nanoparticles with an oleic acid coating were produced by the thermal decomposition of iron chelates. TEM image analysis indicated that the particles have a diameter of $7.2 \mathrm{~nm}$ with a numerical standard deviation of $0.9 \mathrm{~nm}$.

A heterobifunctional PEO was synthesized using a combination of ring opening polymerization and endgroup modification, as portrayed in Scheme 1. Using potassium bis(trimethylsilyl)amide solution as the anionic initiator, we utilized a procedure that has been used previously to create a PEO with a protected amine at one end and a free alcohol at the other.[10, 23]

${ }^{1}$ HNMR confirmed methylene peaks of PEO at $3.56 \mathrm{ppm}$ and the adjacent hydrogen to the protected amide group at $2.75 \mathrm{ppm}$. Using propargyl bromide in the presence of $\mathrm{NaH}$, the free alcohol on the PEO was substituted with propargyl endgroup, indicated by peaks 2.43 and $4.19 \mathrm{ppm}$ of the NMR. Then using $1 \mathrm{M} \mathrm{HCl}$, the trimethylesilyl (TMS) that was protecting our amine was removed, confirmed by the disappearance of the peak. The number average molecular weight was determined by NMR to be 6000 $\mathrm{g} \mathrm{mol}^{-1}$.

The heterobifunctional PEO obtained was now primed to react with the carboxylic acids of PAA, as described in Scheme 2. Using DCC coupling, the amine-PEO-alkyne was reacted with the carboxylic acids of PAA, targeting a 5:1 ratio of PEO to PAA chain.[10, 24] A broad peak at 1.20-2.25 ppm, Figure S1, confirmed the presence of PAA after the reaction. When comparing the ratio of the integrals between the $\alpha$ hydrogen found in the PAA $(25 \mathrm{H})$ and the lone proton of the alkyne terminus of the PEO $(1 \mathrm{H})$ the grafting density of PEO on the PAA was determined to be approximately 6 chains of PEO per PAA molecule. The peaks of the propargyl end group were still present at 2.43 and $4.20 \mathrm{ppm}$. Using FTIR, the modification of PAA was also confirmed by comparing the spectra of PAA before and after it was modified, Figure S2. The peak at $1728 \mathrm{~cm}^{-1}$ corresponds to the carboxyl of the PAA and the peaks at 1648 and $1531 \mathrm{~cm}^{-1}$ are attributed to the carboxyl-amide bond that is found between the carboxyl group of the PAA and the amine of the PEO.

Using EDC coupling, we then randomly modified our PAA-PEO-Alkyne with nitroDOPA, 10:1 ratio. An excess of nitroDOPA was used to ensure there is increased anchoring when modifying the iron oxide nanoparticles. NitroDOPA-PAA-PEO-Alkyne, after purification, was immediately dissolved in chloroform and used in the modification of nanoparticles. Using sonication while slowly adding particles to our particle solution helps facilitate the ligand exchange and ensure maximum surface coverage. The transferring of particles to water was a good indication of a successful modification. Magnetometry conducted on the final product demonstrated superparamagnetic behaviour of the system and saturazation magnetization of $\sim 63 \mathrm{emu} / \mathrm{g}$ of $\mathrm{Fe}_{3} \mathrm{O}_{4}$ was obtained (see supporting information).

Using DLS, surface modification was confirmed by an increase in hydrodynamic radius, Figure S3. The particles were also analysed with FTIR to corroborate the DLS (Figure S2) and confirm that we have the desired surface functionality. As seen in Figure 1, the peak at $3237 \mathrm{~cm}^{-1}$ confirms the presence of the alkyne[24-28] as well as the presence of carboxylic acids from PAA $\left(1711 \mathrm{~cm}^{-1}\right)$ and amide linkages $\left(1643\right.$ and $\left.1561 \mathrm{~cm}^{-1}\right)$ from the nitroDOPA and/or PEO to the PAA. The surface coverage of polymer on the particle was determined by TGA, Figure S4. There was an $81.3 \%$ weight loss, from 372 to $415^{\circ} \mathrm{C}$, from the nitroDOPA-PAA-PEO-Alkyne (9.49 mg).

The particles were then exposed to a series of biological environments to establish if the multianchoring enhances the stability compared to mono-anchored modified particles, as previously reported.[14] PBS is a buffer solution that matches the ion concentrations in the human body and is commonly used in biological research. Each particle solution $(0.1 \mathrm{mg} / \mathrm{mL})$ was titrated with $10,30,50$ and $100 \%$ by volume of $1 \mathrm{~N}$ PBS and the hydrodynamic diameter was observed using DLS. The results shown in Figure 2 show the enhanced stability of the multi-anchored particles compared to the mono-anchored. At $100 \%$ by volume of PBS, for the mono-anchored particles prepared in previous studies,[14] there was an obvious decrease in original particle size and the formation of large clusters greater than $1000 \mathrm{~nm}$. No visible effect on size was observed for the multi-anchored particles. These results are in line with work that has been previously reported pertaining to using multi-anchoring groups to increase stability of magnetic nanoparticles in biological environments.[25-29] It also should be noted that the measured hydrodynamic diameters are in agreement with similar systems with polymer brushes on the order of $4000 \mathrm{~g} \mathrm{~mol}^{-1}$ as well as agrees with calculations that we have presented in previous work.[30][28]

The hydrodynamic diameter of the particles was measured by DLS in a $50 \%$ by volume of PBS near the average human body temperature, $37^{\circ} \mathrm{C}$. In Figure 2 (B), the multi-anchored particles show no change in hydrodynamic diameter in this environment, indicating that the particles do not aggregate in relatively high ionic strength environments. The mono-anchored size distribution, although colloidally 
stable in the same ionic environment, broadens with an increase in temperature, suggesting aggregation. The multiple nitroDOPA groups increase the amount of interactions with the surface of the iron oxide nanoparticles keeping the steric interactions intact that are involved in keeping the particles discrete and colloidally stable. This multidentate approach has been demonstrated to be successful.[10, 25, 31]

The stability of the multi-anchored nanoparticles was further studied in a protein rich environment by submitting them to a $50 \%$ solution of FBS in DI water and measuring their hydrodynamic diameter. As observed in Table 1, there is no significant increase in hydrodynamic diameter, for either the mono or multi-anchored nanoparticles, after adding FBS. The temperature was then increased to $70{ }^{\circ} \mathrm{C}$ to submit the particles to an accelerated test of their stability in this challenging environment. Initially, little discernable difference in hydrodynamic diameter from the previous measurements at room temperature was observed. After 24 hours at $70{ }^{\circ} \mathrm{C}$, the mono-anchored nanoparticles doubled in hydrodynamic diameters due to the protein interactions with the polymer brushes on the surface. The proteins, causing the particles to cluster and lose their colloidal stability, are displacing the mono-anchored polymer. There was no clustering observed with the multi-anchored nanoparticles because of the multiple nitroDOPA groups interacting with the surface of the magnetic nanoparticles. These multiple interactions are robust enough to decrease the displacement of the polymer surface with the proteins.

The cytotoxicity of the multi-anchored nanoparticles was determined on mouse primary fibroblast (L929) cell lines. To ensure there are no negative biological effects of the nanoparticles, MTS assays were performed with seven different concentrations of iron, Figure 3. The cells incubated for 48 hours showed no statistically significant difference in viability between the different concentrations and the control $(\mathrm{p}>$ 0.05, One-Way ANOVA test).

The particle surface was then modified with Cy5.5 azide. This a near-infrared (NIR) dye that is used for in vivo imaging.[29, 32] The click reaction took place in water using copper sulfate and sodium ascorbate to drive the reaction and later the particles were dialyzed in DI water for 3 days to ensure removal of any excess dye or copper catalyst. In Figure 4, photoluminescence confirms the modification with an emission at $701 \mathrm{~nm}$. The photoluminescence of multi-anchored particles was measured to show that the particles were not adding to the photoluminescence.

Once the particles were modified with Cy5.5, they were then used as a tool to monitor the location of the magnetic nanoparticles optically in a Legionella pneumophila biofilm. The modified magnetic nanoparticles were incubated with the biofilm for 48 hours and then imaged with a Leica SR GSD 3D, Super Resolution Ground State Depletion 3D Microscope (Leica Microsystems, Buffalo Grove, IL). In the $3 \mathrm{D}$ reconstruction of the control biofilm and biofilms treated with Cy5.5 coated magnetic nanoparticles, Figure 5A and B respectively, the particles can be observed in association with the bacteria within the biofilm. Syto 11 stains nucleic acid, but does not provide definition of the cell wall. NP localization with the bacteria after numerous washing steps is indicative of binding of the NPs with either the bacteria or the biofilm matrix. The manner of association between the bacteria and NPs is still unknown; however, future GSD studies including using fluorescent stains for cell membrane components in order to highlight the bacterial cell walls, will allow us to decipher if the nanoparticles are interacting directly with the bacteria or the matrix. This is in line with previous studies that have quantified the loss of biomass due to nanoparticle exposure.[8, 32, 33] Traditionally, biofilms have been characterized using confocal microscopy and quantification of their biomass, surface roughness, thickness, and other physical parameters.

In tradition confocal microscopy it is challenging to image individual nanoparticles, as their size is below the resolution limit of the technique, which is approximately $200 \mathrm{~nm}$. When imaged using traditional confocal microscopy, biofilms treated with fluorescent NPs show the biofilm with a haze of fluorescence representing the NPs, as they are too small to be sufficiently resolved. By using super-resolution microscopy, which has a much higher resolving power than traditional confocal microscopy (down to approximately $20 \mathrm{~nm}$ laterally), individual nanoparticles may be visualized. This allows direct, 3D observation of single nanoparticles with individual bacteria within a biofilm.

While it is well known that nanoparticles of various composition are able to disrupt bacterial biofilms, the mechanisms behind this disruption are not known. \{Raftery, 2012 \#2677\} \{Laurent, 2008 \#2654\}\{Raftery, $2014 \# 1397\}$ Future investigations using tagged NPs and GSD super resolution microscopy will help to elucidate the manner of association between NPs and the bacteria within biofilms. Fluorescent stains for bacterial cell wall components, as well as immuno-fluorescent labeling of known bacterial adhesion proteins, may be utilized to determine if there is a direct interaction between the NPs and the bacteria. Additionally, the biofilm matrix may be fluorescently stained to determine if the NPs are interacting directly with the matrix. The high resolution imaging available with 3D GSD will help 
determine the spatial distribution of NPs in the biofilm, as well as their manner of interaction with either the bacteria directly or with the biofilm matrix. This information will help to determine if the disruption of bacterial biofilms is due to a direct interaction between the NPs and the bacteria or the biofilm matrix, or if it is due instead to a spatial disruption that renders the surface non-compatible for biofilm growth. Once the manner of NP association with biofilms is better understood, NPs may be able to be utilized in studies of biofilm life cycle, stability and persistence. The disruption of the biofilms was characterized by observing the change in biomass, thickness, and roughness compared to a control that was not treated. There was a statistically significant decrease in biomass (Figure S7) and thickness (Figure S8). We also observed an almost $40 \%$ in roughness compared to the control, Figure S9. The nanoparticle are disrupting the matrix of the biofilm, hindering its viability.

\title{
Conclusions
}

By adapting a multidentate approach for surface functionalization, these magnetic nanomaterials provide a versatile platform for additional modification. Challenges with PBS and FBS demonstrate no measureable particle aggregation suggesting the colloidal stability of these materials in biological media. Cell viability testing demonstrated no significant decrease up to $63 \mathrm{mg} / \mathrm{mL}$. Labelling of this platform with Cy5.5 established the ability of these materials to be used also as imaging agents in biofilms. The materials discussed in above are not limited to the imaging of biofilms, they have potential in other areas of research including multimodal therapeutic and diagnostic agents that can improve upon current cancer[33, 34], antibiotic treatments,[15] as well as the remediation of biofilms,[8, 32] is bright.

\section{Acknowledgements}

The authors would like to thank the Clemson Light Imaging Facility and Dr. Robyn Schlicher, Leica Microsystems, for assistance with super resolution sample preparation and microscopy. In addition, the authors thank Leica Microsystems for the use of the Leica SR GSD 3D microscopy system through their ongoing partnership with the Clemson Light Imaging Facility. This work was performed, in part, at the Center for Integrated Nanotechnologies, an Office of Science User Facility operated for the U.S. Department of Energy (DOE) Office of Science. Sandia National Laboratories is a multi-program laboratory managed and operated by Sandia Corporation, a wholly owned subsidiary of Lockheed Martin Corporation, for the U.S. Department of Energy's National Nuclear Security Administration under contract DE-AC04-94AL85000. Special thanks for funding from the National Science Foundation under grant numbers: ECC1062873, CMMI-1057633, and CMMI-1130819

\author{
Notes and references \\ ${ }^{a}$ Clemson University, School of Materials Science and Engineering and Center for Optical Materials \\ Science and Engineering Technologies (COMSET), 91 Technology Drive, Anderson, SC 29625, USA \\ ${ }^{b}$ University of Wisconsin-Madison, Materials Science and Engineering, 1509 University Avenue, Madison \\ WI 53706, USA \\ ${ }^{c}$ Clemson University, Department of Biological Sciences, 132 Long Hall, Clemson, SC 29634, USA \\ ${ }^{d}$ Clemson University Institute of Environmental Toxicology (CU-ENTOX), 509 Westinghouse Road, \\ Pendleton, SC 29670, USA \\ ${ }^{e}$ Clemson University, Light Imaging Facility, 190 Collings Street, Clemson, SC 29634, USA \\ ${ }^{\mathrm{f}}$ Sandia National Laboratories, Albuquerque, NM 87185, USA
}

\section{References}

[1] L. Babes, B. Denizot, G. Tanguy, J.J. Le Jeune, P. Jallet, Journal Of Colloid And Interface Science 212 (1999) 474.

[2] S. Balivada, R. Rachakatla, H. Wang, T.N. Samarakoon, R. Dani, M. Pyle, F.O. Kroh, B. Walker, X. Leaym, O.B. Koper, M. Tamura, V. Chikan, S.H. Bossmann, D.L. Troyer, BMC Cancer 10 (2010) 119.

[3] J.W.M. Bulte, D.L. Kraitchman, NMR in Biomedicine 17 (2004) 484. 
[4] Q.A. Pankhurst, J. Connolly, S. Jones, J. Dobson, Journal of Physics D: Applied Physics 36 (2003) R167.

[5] M. Kuhara, H. Takeyama, T. Tanaka, T. Matsunaga, Analytical Chemistry 76 (2004) 6207.

[6] K.E. McCloskey, J.J. Chalmers, M. Zborowski, Analytical Chemistry 75 (2003) 6868.

[7] R. Stone, T. Willi, Y. Rosen, O.T. Mefford, F. Alexis, Therapeutic Delivery 2 (2011) 815.

[8] T.D. Raftery, P. Kerscher, A.E. Hart, S.L. Saville, B. Qi, C.L. Kitchens, O.T. Mefford, T.L. Mcnealy, Nanotoxicology 8 (2014) 477.

[9] S.L. Saville, R.C. Stone, B. Qi, O.T. Mefford, Journal of Materials Chemistry 22 (2012) 24909.

[10] H.B. Na, G. Palui, J.T. Rosenberg, X. Ji, S.C. Grant, H. Mattoussi, ACS nano 6 (2012) 389.

[11] M. Das, D. Bandyopadhyay, D. Mishra, S. Datir, P. Dhak, S. Jain, T.K. Maiti, A. Basak, P. Pramanik, Bioconjugate Chemistry 22 (2011) 1181.

[12] Z. Li, Y. Chau, Bioconjugate Chemistry 20 (2009) 780.

[13] P. Avti, D. Maysinger, A. Kakkar, Molecules 18 (2013) 9531.

[14] R.C. Stone, B. Qi, D. Trebatoski, R. Jetti, Y.P. Bandera, S.H. Foulger, O.T. Mefford, Journal of Materials Chemistry B 2 (2014) 4789.

[15] Y.S. Raval, R. Stone, B. Fellows, B. Qi, G. Huang, O.T. Mefford, T.-R.J. Tzeng, Nanoscale 7 (2015) 8326.

[16] S. Sun, H. Zeng, D. Robinson, S. Raoux, P. Rice, S. Wang, G. Lis, J. Am. Chem. Soc 126 (2004) 273.

[17] Z. Li, S. Yin, L. Cheng, K. Yang, Y. Li, Z. Liu, Advanced Functional Materials 24 (2013) 2312.

[18] R. Torres Martin de Rosales, R. Tavaré, A. Glaria, G. Varma, A. Protti, P.J. Blower, Bioconjugate Chemistry 22 (2011) 455.

[19] M.A. Daniele, M.L. Shaughnessy, R. Roeder, A. Childress, Y.P. Bandera, S. Foulger, ACS nano 7 (2012) 203.

[20] S. Corr, Y. Gun'ko, A. Douvalis, M. Venkatesan, pubs.acs.org (2008).

[21] S. Prijic, J. Scancar, R. Romih, M. Cemazar, V.B. Bregar, A. Znidarsic, G. Sersa, The Journal of Membrane Biology 236 (2010) 167.

[22] Y. Kim, Y. Nagasaki, K. Kataoka, M. Kato, M. Yokoyama, T. Okano, Y. Sakurai, Polymer Bulletin 33 (1994) 1.

[23] J.K. Tessmar, A.G. Mikos, A. Göpferich, Biomacromolecules 3 (2002) 194.

[24] X. Fan, B. Huang, G. Wang, J. Huang, Macromolecules 45 (2012) 3779.

[25] S.L. Saville, R.C. Stone, B. Qi, O.T. Mefford, Journal of Materials Chemistry 22 (2012) 24909.

[26] J.D. Goff, P.P. Huffstetler, W.C. Miles, N. Pothayee, C.M. Reinholz, S. Ball, R.M. Davis, J.S. Riffle, Chemistry Of Materials 21 (2009) 4784.

[27] W.C. Miles, J.D. Goff, P.P. Huffstetler, C.M. Reinholz, N. Pothayee, B.L. Caba, J.S. Boyd, R.A. Davis, J.S. Riffle, Langmuir 25 (2009) 803.

[28] L.A. Harris, J.D. Goff, A.Y. Carmichael, J.S. Riffle, J.J. Harburn, T.G. St Pierre, M. Saunders, Chemistry Of Materials 15 (2003) 1367. 
[29] S.K. Yen, D. Jańczewski, J.L. Lakshmi, S.B. Dolmanan, S. Tripathy, V.H.B. Ho, V. Vijayaragavan, A. Hariharan, P. Padmanabhan, K.K. Bhakoo, T. Sudhaharan, S. Ahmed, Y. Zhang, S. Tamil Selvan, ACS nano 7 (2013) 6796.

[30] S.L. Saville, R. Woodward, M. House, A. Tokarev, J. Hammers, B. Qi, J.A. Shaw, M. Saunders, R. Versani, T.G. St Pierre, O.T. Mefford, Nanoscale 5 (2013) 2152.

[31] M.H. Stewart, K. Susumu, B.C. Mei, I.L. Medintz, J.B. Delehanty, J.B. BlancoCanosa, P.E. Dawson, H. Mattoussi, Journal Of The American Chemical Society 132 (2010) 9804.

[32] T.D. Raftery, H. Lindler, T.L. McNealy, Microbial Ecology 65 (2012) 496.

[33] S. Laurent, D. Forge, M. Port, A. Roch, C. Robic, L. Vander Elst, R.N. Muller, Chemical Reviews 108 (2008) 2064.

[34] L.W. Qu, P.G. Luo, S. Taylor, Y. Lin, W.J. Huang, N. Anyadike, T.R.J. Tzeng, F. Stutzenberger, R.A. Latour, Y.P. Sun, J Nanosci Nanotechno 5 (2005) 319. 


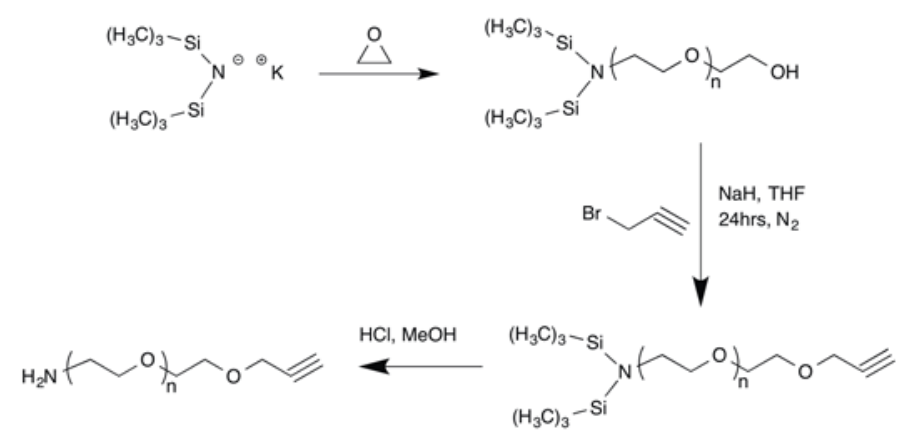

Scheme 1. Synthesis and endgroup modification of the heterobifunctional PEO, AminePEO-Alkyne<smiles>CCC(CC(C)C)C(=O)O</smiles>
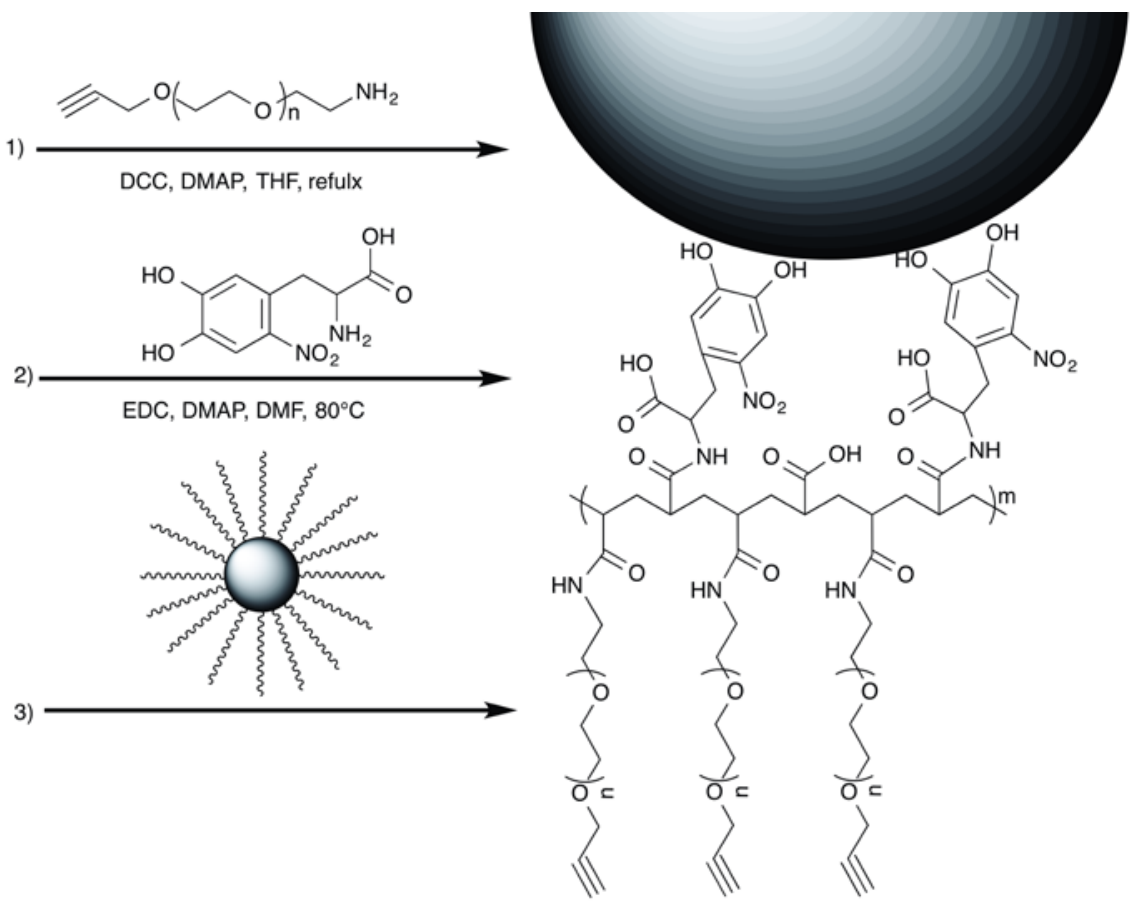

Scheme 2. Synthetic design of NitroDOPA-PAA-PEO-Alkyne and particle modification 


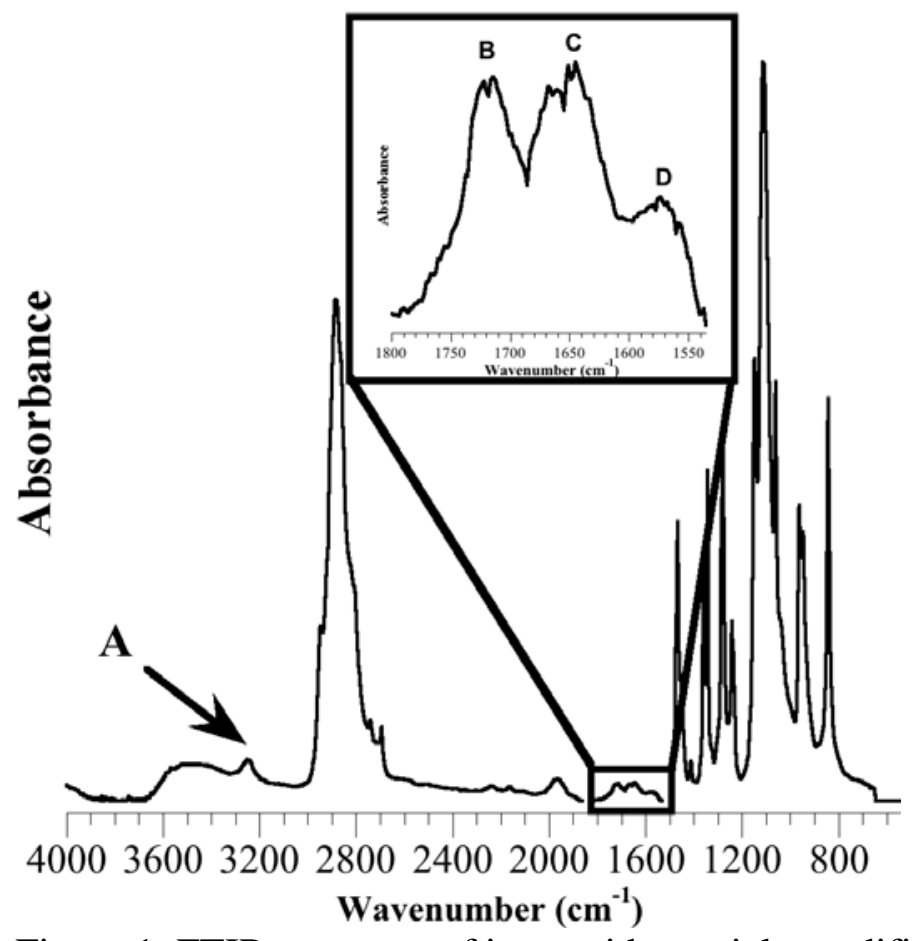

Figure 1. FTIR spectrum of iron oxide particle modified with nitroDOPA-PAA-PEOAlkyne. A. Alkyne group on the surface of the particle $\left(3237 \mathrm{~cm}^{-1}\right)$. B. Carboxylic acid groups of PAA $\left(1711 \mathrm{~cm}^{-1}\right)$. C and D. Amide linkages to PAA $\left(1643\right.$ and $\left.1561 \mathrm{~cm}^{-1}\right)$.
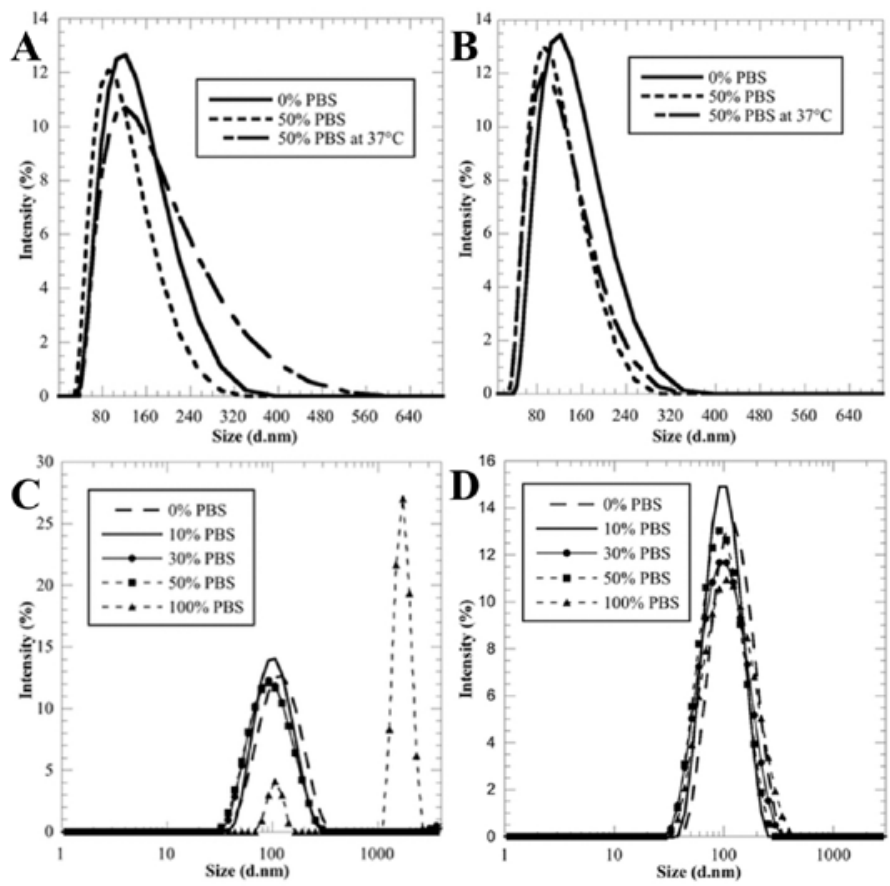

Figure 2. Stability studies comparing mono and multi-anchored particles. A) DLS of mono-functional particles in $50 \%$ by volume PBS at $37^{\circ} \mathrm{C}$; B) DLS of multi-functional particles in $50 \%$ by volume PBS at $37^{\circ} \mathrm{C}$; C) DLS of mono-anchored particles titrated 
with PBS from 0-100\% by volume; D) DLS of multi-anchored particles titrated with PBS from $0-100 \%$ by volume.

Table 1. Presents the results of the time dependence studies done in 50\% FBS for both the mono-anchored and multi-anchored magnetic nanoparticle complexes, where *average hydrodynamic diameter over 24 hours

\begin{tabular}{|l|c|c|}
\hline & $\begin{array}{c}\text { Mono-anchored } \\
(\mathbf{d} . n m)\end{array}$ & $\begin{array}{c}\text { Multi-anchored } \\
\text { (d.nm) }\end{array}$ \\
\hline DI Water at $\mathbf{2 5}^{\circ} \mathbf{C}$ time $=\mathbf{0 ~ s}$ & 119 & 106 \\
\hline $\mathbf{5 0 \%}$ FBS at $\mathbf{2 5}^{\circ} \mathbf{C}$ time $=\mathbf{0 ~ s}$ & 121 & 101 \\
\hline $\mathbf{5 0 \%}$ FBS at $\mathbf{7 0}^{\circ} \mathbf{C}$ time $=\mathbf{0 ~ s}$ & 122 & 108 \\
\hline $\mathbf{5 0 \%}$ FBS at $\mathbf{7 0}^{\circ} \mathbf{C}$ time $=\mathbf{2 4}$ hours* & $204 \pm 30$ & $96 \pm 22$ \\
\hline
\end{tabular}

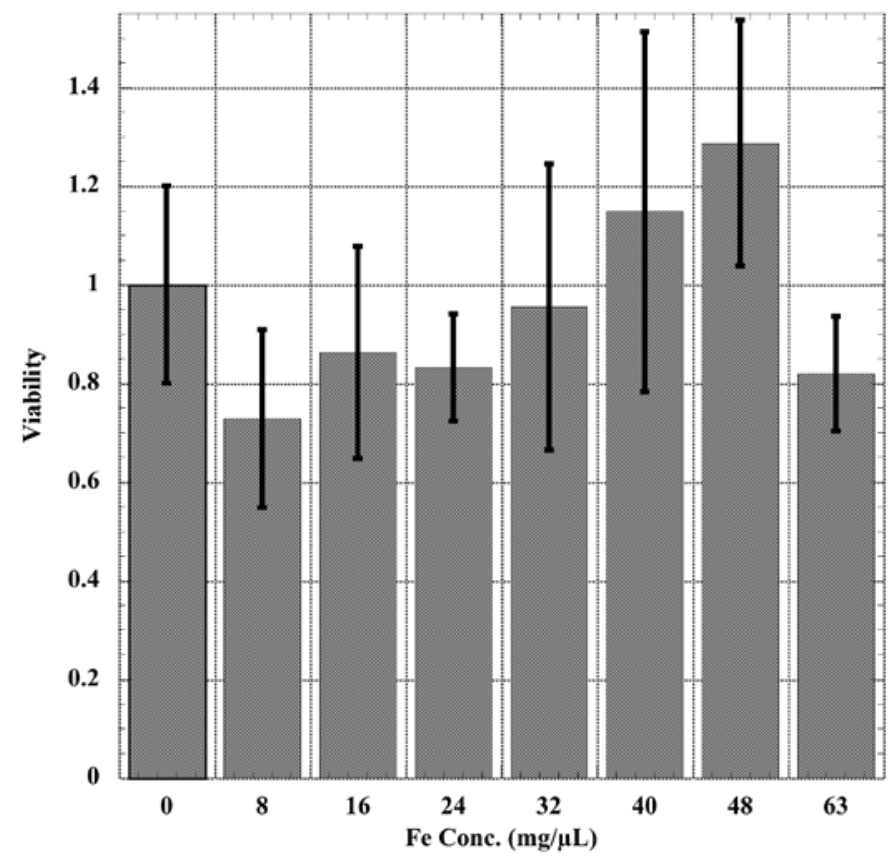

Figure 3. Cell viability of cells exposed to multi-anchored particles at different concentrations after 48 hours of incubation in L929 cell line $(n=3)$. 


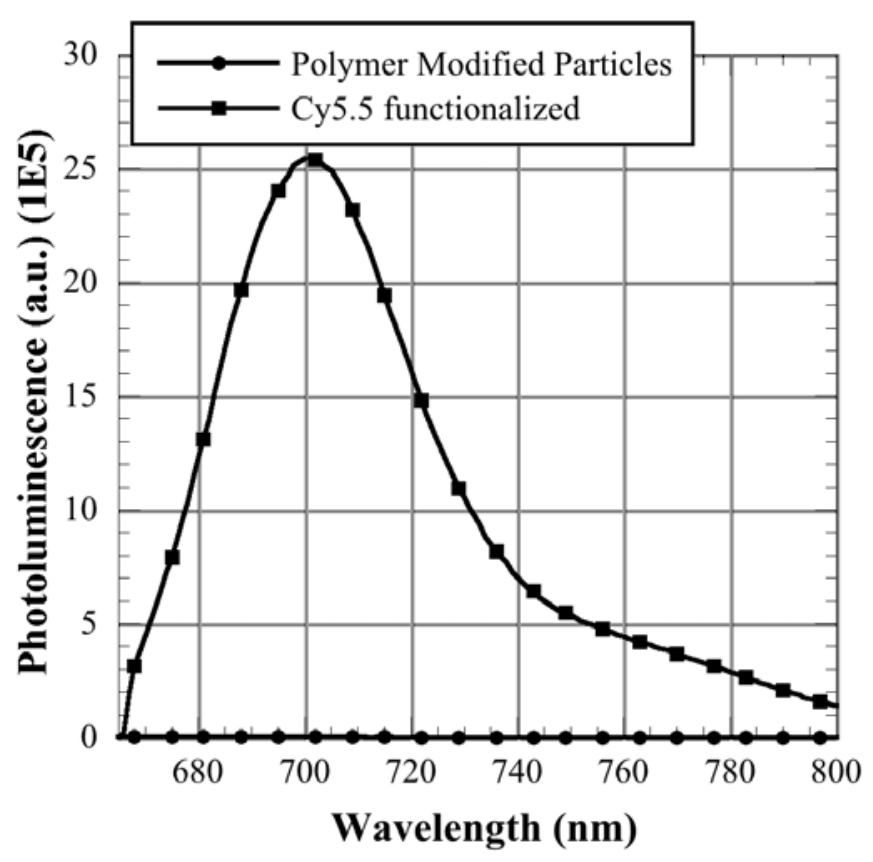

Figure 4. Photoluminescence of multi-anchored particles before and after 'clicking' on azido-Cy5.5.

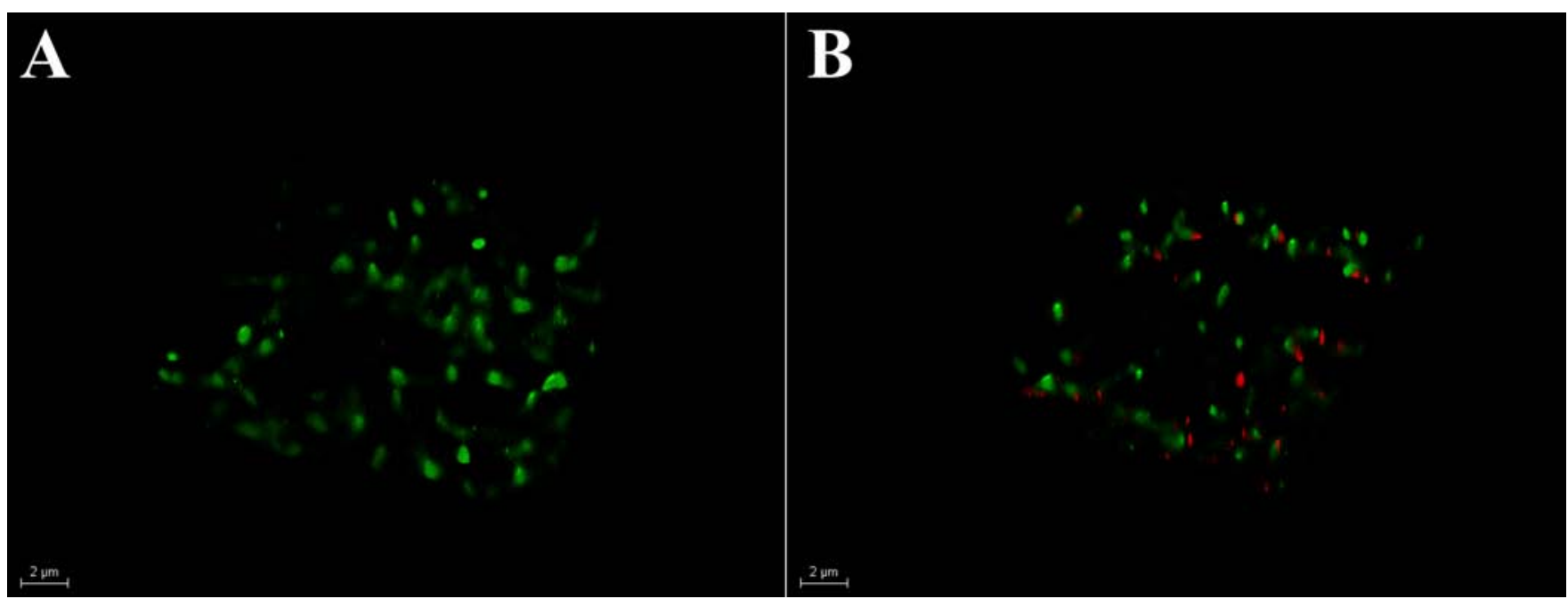

Figure 5. A) 3D micrograph of Legionella pneumophila biofilm control. B) 3D micrograph of Legionella pneumophila biofilm (green) incubated with multi-anchored nanoparticles modified with Cy5.5 (red). Scale bars in each image are $2 \mu \mathrm{m}$. 